\title{
Aspectos Sociais na Formação dos Hábitos Alimentares de Crianças
}

\author{
Social Aspects in the Formation of Children's Eating Habits
}

Aspectos sociales en la formación de hábitos alimentarios infantiles

\begin{abstract}
Mirian Lene da Roz Vieira ${ }^{1}$, Jennyfer Carolini Santos de Oliveira ${ }^{1}$, Ana Paula de Queiroz Mello ${ }^{1 *}$.
\end{abstract}
\begin{abstract}
RESUMO
Objetivo: Identificar aspectos sociais que possam influenciar na formação dos hábitos alimentares de crianças. Métodos: Foram realizadas pesquisas incorporando as bases de dados bibliográficas: Scientific Electronic Library Scielo, Biblioteca virtual em saúde Bireme, MEDLINE / Pubmed. Como critérios de inclusão foram definidos artigos científicos originais publicados na íntegra nos últimos 5 anos, em português e inglês. A busca teve como descritores as palavras: obesidade infantil, transição nutricional, hábito alimentar, família, nutrição, políticas públicas, educação alimentar e nutricional. Resultados: A família, a escola e a mídia desempenham importante papel na formação do hábito alimentar das crianças que refletem no seu estado nutricional. Considerações finais: É evidente a relação do consumo alimentar dos pais e/ou familiares com o estado nutricional dos filhos, e sua importância na formação do hábito alimentar da criança.
\end{abstract}

Palavras-chave: Obesidade Infantil, Hábito Alimentar, Família.

\begin{abstract}
Objective: Identify social aspects that may influence the formation of children's eating habits. Methods: Researches were carried out incorporating the bibliographic databases: Scientific Electronic Library Scielo, Bireme virtual health library, MEDLINE / Pubmed. As inclusion criteria were defined original scientific articles published in full in the last 5 years, in Portuguese and English. The search had as keywords the words: childhood obesity, nutritional transition, food habits, family, nutrition, public policies, food and nutritional education. Results: The family, the school and the media play an important role in the formation of children's eating habits, which reflect on their nutritional status. Final considerations: It is evident the relation of the food consumption of the parents and / or relatives with the nutritional state of the children, and its importance in the formation of the alimentary habits of the child.
\end{abstract}

Key words: Childhood Obesity, Eating Habits, Family.

\section{RESUMEN}

Objetivo: Identificar aspectos sociales que pueden influir en la formación de los hábitos alimenticios de los niños. Métodos: Se realizaron investigaciones incorporando las bases de datos bibliográficas: Scientific Electronic Library Scielo, Biblioteca virtual en salud Bireme, MEDLINE / Pubmed. Los criterios de inclusión fueron artículos científicos originales publicados en su totalidad en los últimos cinco años, en portugués y en Inglés. La búsqueda tuvo como descriptores las palabras: obesidad infantil, transición nutricional, hábito alimentario, familia, nutrición, políticas públicas, educación alimentaria y nutricional. Resultados: La familia, la escuela y los medios de comunicación desempeñan un importante papel en la formación del hábito alimentario de los niños que reflejan en su estado nutricional. Consideraciones finales: Es evidente la relación del consumo alimentario de los padres y / o familiares con el estado nutricional de los hijos, y su importancia en la formación del hábito alimentario del niño.

Palabras clave: Obesidad Infantil, Hábito Alimenticio, Familia.

${ }^{1}$ Centro Universitário São Camilo, São Paulo - SP. *E-mail: apqmello@gmail.com

SUBMETIDO EM: 4/2019 | ACEITO EM: 5/2019 | PUBLICADO EM: 10/2019

REAS/EJCH | Vol.Sup.33 | e809 | DOI: https://doi.org/10.25248/reas.eXX.2019 Página 1 de 7 


\section{INTRODUÇÃO}

Segundo estudos das últimas décadas, uma crescente mudança vem ocorrendo no estilo de vida da população mundial, onde há uma maior ingestão de alimentos industrializados e diminuição na prática de atividades físicas. No Brasil, tem-se observado uma mudança no perfil nutricional, com aumento significativo das doenças relacionadas à obesidade (FERNANDES CS et al., 2017). Esse processo é caracterizado como transição nutricional, e tem despertado a necessidade de novos enfoques explicativos e intervencionistas no campo da Nutrição, com destaque no desenvolvimento de estratégias eficazes de prevenção da obesidade (NASCIMENTO et al., 2016).

De acordo com a Organização Mundial da Saúde (OMS), a obesidade infantil é um dos mais sérios problemas de saúde pública do século atual, sendo considerado global e afetando principalmente países de baixa e média renda em áreas urbanas. A prevalência da doença tem aumentado de modo alarmante e, em 2010, o número de crianças com sobrepeso foi de mais de 42 milhões, sendo 35 milhões residentes em países em desenvolvimento (WHO, 2014).

Com base nesses estudos, a alta taxa de excesso de peso é considerada preditor importante no desenvolvimento da obesidade e das doenças crônicas não transmissíveis (DCNTs) na vida adulta. Esse resultado reforça os achados de maior consumo de alimentos ricos em gorduras e açúcares, como biscoitos recheados, doces e refrigerantes (SPARRENBERGER $\mathrm{K}$ et al., 2015).

De acordo com o Instituto Brasileiro de Geografia e Estatística (IBGE), os padrões de consumo alimentar dos brasileiros mudaram. A Pesquisa de Orçamento Familiar (POF), de 2008/2009, mostra que a aquisição e consumo de alimentos fora de casa em contraste com a pesquisa anterior em 2002/03 subiu de 20\% para $31,1 \%$, aproximadamente (IBGE, 2010). O perfil epidemiológico e os problemas de saúde da população brasileira mudaram ao longo do último século no Brasil, que refletiram no aumento da expectativa de vida da população no geral. No entanto, de forma concomitante, houve aumento do excesso de peso corporal e da morbidade e mortalidade por DCNTs. Atualmente no Brasil, mais de $50 \%$ dos adultos têm excesso de peso, $14,8 \%$ são tabagistas e $79,8 \%$ não consomem pelo menos cinco ou mais porções de frutas, verduras ou legumes por dia (FLORINDO AA et al., 2016).

O crescimento da obesidade é alarmante, e considerada já um enorme problema de saúde pública e uma epidemia mundial. Nesse sentido, fazem-se necessárias reflexões sobre agentes considerados como fatores de risco para o desenvolvimento da obesidade infantil, abordando-se também a carência de políticas públicas efetivas para prevenção e tratamento dessa doença. E quando se trata da obesidade infantil, deve-se abordar de maneira ampla e não apenas o estado nutricional da criança, mas também aspectos que abrangem seu contexto familiar e o ambiente no qual está inserida (DURÉ ML et al., 2015).

Devido às mudanças no perfil familiar por conta da inserção da mulher no mercado de trabalho, nota se uma elevação no número de crianças na fase pré escolar em creches gratuitas nas médias e grandes cidades. Etapa a qual se inicia a formação dos hábitos, práticas, escolhas e preferências alimentares, modo de vida da família e as condições que favorecem o consumo de determinado alimento tais como: disponibilidade, estado emocional, cultura, relações sociais, dentre outros. Observa se assim que a influencia dos pais em relação ao ambiente familiar, desempenha papel determinante no desenvolvimento destes indivíduos e estes fatores estão diretamente ligados à formação dos hábitos futuros (BENTO IC et al, 2015).

Refeições familiares frequentes estão associadas a melhores desfechos sociais para crianças e adolescentes. Há uma relação positiva entre refeições familiares frequentes no qual há um aumento da autoestima e compromisso com a aprendizagem dos filhos. O que se sabe sobre as barreiras que existem para ter refeições familiares frequentes é que os pais são muito ocupados e apresentam pouca disponibilidade de horários (HARRISON ME et al., 2015). A criança desde muito cedo aprende a fazer suas escolhas com base no que observa nas atitudes dos seus familiares, especialmente dos pais, sendo assim, é provável que a criança venha a querer ingerir o mesmo alimento consumido por eles. Desta forma, as escolhas inadequadas por parte dos que vivem no mesmo ambiente familiar que as crianças irão influenciar as suas escolhas, resultando em uma má alimentação e excesso de peso (LINHARES FMM et al., 2018). Segundo 
recente estudo, a maior atenção tem sido dada ao impacto negativo do consumo de alimentos com baixa qualidade nutricional, que fortemente estão associados à saúde atual e aos padrões alimentares futuros, e este consumo pode estar relacionado à disponibilidade domiciliar dos alimentos (JAIME PC et al., 2017).

Em relação às complicações clínicas da obesidade, esta está envolvida no desequilíbrio energético entre ingestão e gasto, sendo influenciado por vários fatores neuroendócrinos, como as adipocitocinas, proteínas produzidas pelo tecido adiposo que é considerado um órgão endócrino. A insulina também participa e é um hormônio com importantes funções metabólicas, tais como, promover adequada captação periférica de glicose, suprimir a gliconeogênese hepática e inibir a produção de lipoproteína de muito baixa densidade (TAVARES GT et al., 2016). A resistência insulínica é definida como a ineficiência da insulina plasmática, em concentrações normais. Na faixa etária pediátrica, a obesidade parece ser importante gatilho para a resistência insulínica, o que torna as crianças obesas um grupo de risco, visto que essa resistência tem papel fisiopatológico importante na gênese da doença cardiovascular aterosclerótica. O excesso de peso na infância é considerado o principal fator para que a obesidade se desenvolva e estenda-se até a fase adulta. Estudos evidenciam que o processo aterosclerótico se inicia na infância, no qual são influenciados por fatores hereditários e ambientais, evoluindo clinicamente na idade adulta (TAVARES GT et al., 2016).

O acúmulo de tecido adiposo, principalmente na região abdominal, é fundamental para o desencadeamento da síndrome metabólica (SM). Existe uma associação entre o tecido adiposo e as principais células inflamatórias, levando a um aumento da produção de mediadores inflamatórios e a maior liberação de ácidos graxos livres, cujos efeitos se fazem sentir tanto nas células beta das ilhotas de Langerhans e seus receptores como na parede vascular (CARVALHO ARM et al., 2017). Estudos em crianças e adolescentes comprovam como esses fenômenos começam precocemente. Apesar de que na faixa etária mais precoce, a morbidade não é frequente, a presença de alterações metabólicas (resistência à insulina, dislipidemia, hipertensão, alterações trombogênicas, hiperuricemia) na infância e adolescência pode contribuir para alta taxa de morbimortalidade na vida adulta por doenças cardiovasculares (CARVALHO ARM et al., 2017; JAIME PC et al., 2017).

Considerando a obesidade infantil sob a óptica dos dados epidemiológicos, dos fatores de risco e suas complicações, se faz necessário entendermos os aspectos sociais que influenciam a formação do hábito alimentar nas crianças.

\section{MÉTODOS}

Para responder essa questão, foram realizadas pesquisas incorporando as bases de dados bibliográficas: Scientific Electronic Library Scielo, Biblioteca virtual em saúde Bireme, MEDLINE / Pubmed. Como critérios de inclusão foram definidos artigos científicos originais publicados na íntegra nos últimos 5 anos, em português e inglês. Além disso, artigos que envolviam crianças de 0 a 10 anos de idade, com apresentação de dados socioeconômicos, nível de escolaridade dos pais e cuidadores, estado nutricional, influência da mídia, alimentação dos pais e/ou cuidadores, além de estratégias em saúde pública.

A busca teve como descritores as palavras: obesidade infantil, transição nutricional, hábito alimentar, família, nutrição, políticas públicas, educação alimentar e nutricional. A partir dos descritores citados acima, primeiramente, foram encontrados 6.372 artigos relacionados à obesidade infantil que foram reduzidos ao adicionarem os demais na base de dados MEDLINE/ Pubmed. Delimitando o período da busca 2014 a 2019, realizou-se a leitura na íntegra, para avaliar quais revisões estavam dentro do escopo do presente trabalho, no qual, foram selecionados 19 artigos para a elaboração deste manuscrito, e apenas 8 foram utilizados seguindo o objetivo principal do trabalho.

\section{RESULTADOS E DISCUSSÃO}

De acordo com os resultados da pesquisa do presente trabalho, foram selecionados 8 artigos que foram discutidos na luz do entendimento da influência dos aspectos sociais na formação do hábito alimentar e, consequentemente, do estado nutricional das crianças (Quadro 1). 
Quadro 1 - Artigos selecionados para elaboração deste manuscrito com base no tema proposto.

\begin{tabular}{|c|c|c|}
\hline Autor (Ano) / Pais & Objetivo & Conclusão \\
\hline Bento IC et al. (2015) / Brasil & $\begin{array}{l}\text { Conhecer as percepções sobre } \\
\text { alimentação saudável dos responsáveis } \\
\text { por pré-escolares de uma Creche em Belo } \\
\text { Horizonte/MG. }\end{array}$ & $\begin{array}{l}\text { O grupo estudado tem noção do que é uma alimentação saudável, no entanto, não } \\
\text { é refletida em seus discursos, que evidenciam uma prática alimentar inadequada. } \\
\text { Dificuldades em ter uma alimentação saudável estão relacionadas com recursos } \\
\text { financeiros, tempo e hábito inadequado. }\end{array}$ \\
\hline Duré ML et al. (2015) / Brasil & $\begin{array}{l}\text { Discutir a problemática da obesidade } \\
\text { infantil e a sua relação com mídia } \\
\text { audiovisual, relações familiares e } \\
\text { instituições acadêmicas. }\end{array}$ & $\begin{array}{l}\text { Necessidade de criação de políticas públicas que normatizem propagandas } \\
\text { destinadas ao público infantil. Constatou-se que a escola exerce papel } \\
\text { fundamental na prática de hábitos alimentares saudáveis. E, a família tem o papel } \\
\text { de mediar e controlar tais ações. }\end{array}$ \\
\hline $\begin{array}{l}\text { Fechine ADL et al. (2015) / } \\
\text { Brasil }\end{array}$ & $\begin{array}{l}\text { Identificar as percepções de pais e } \\
\text { professores sobre a influência de alimentos } \\
\text { industrializados na saúde infantil. }\end{array}$ & $\begin{array}{l}\text { Existem diversos fatores que podem influenciar o consumo de alimentos } \\
\text { industrializados pelas crianças. Evidencia-se a necessidade de intervenções que } \\
\text { tenham como foco a escola e a família, para prevenir o uso indiscriminado destes } \\
\text { alimentos. }\end{array}$ \\
\hline Flattum C et al. (2015) / EUA & $\begin{array}{l}\text { Reduzir o comportamento sedentário das } \\
\text { crianças, e prevenir a obesidade. }\end{array}$ & $\begin{array}{l}\text { O Programa HOME Plus sugere criação de programas focados na família e mostra } \\
\text { que esta abordagem pode representar uma estratégia eficaz para promover a } \\
\text { saúde através das refeições e prevenir a obesidade entre os jovens pré- } \\
\text { adolescentes. }\end{array}$ \\
\hline $\begin{array}{l}\text { Linhares FMM et al. (2016) / } \\
\text { Brasil }\end{array}$ & $\begin{array}{l}\text { Identificar a relação da influência dos pais } \\
\text { com a educação alimentar dos filhos. }\end{array}$ & $\begin{array}{l}\hat{E} \text { importante que se tenham conhecimento dos fatores causais da obesidade } \\
\text { infantil, e que os pais sejam conhecedores e promovam soluções que minimizem } \\
\text { os riscos da criança desenvolver tal doença. }\end{array}$ \\
\hline Lins ZMB et al. (2015) / Brasil & $\begin{array}{l}\text { Analisar a compreensão que mães e pais } \\
\text { têm sobre o seu papel na criação dos filhos, } \\
\text { e identificar as influências externas no } \\
\text { processo. }\end{array}$ & $\begin{array}{l}\text { As mães foram consideradas responsáveis pela educação e carinho dos filhos, e } \\
\text { os pais, responsáveis pelo exercício da autoridade. Como influências externas, os } \\
\text { pais e mães ressaltaram os avós, a escola e a TV. Estes resultados contribuem } \\
\text { para uma melhor compreensão das atribuições de papéis parentais, e para a } \\
\text { percepção das influências externas que interferem na educação dos filhos. }\end{array}$ \\
\hline $\begin{array}{l}\text { Relvas GRB et al. (2018) / } \\
\text { Brasil }\end{array}$ & $\begin{array}{l}\text { Analisar a prevalência do consumo de } \\
\text { alimentos ultraprocessados entre crianças } \\
\text { com menos de um ano e identificar os } \\
\text { fatores associados. }\end{array}$ & $\begin{array}{l}\text { Foi encontrado consumo elevado de alimentos ultraprocessados entre as crianças } \\
\text { com menos de um ano. A situação socioeconômica materna e o tempo da primeira } \\
\text { consulta da criança na unidade de atenção primária à saúde foram associados à } \\
\text { prevalência de consumo de alimentos ultraprocessados. }\end{array}$ \\
\hline $\begin{array}{c}\text { Soares BR et al. (2017) / } \\
\text { Brasil }\end{array}$ & $\begin{array}{l}\text { Identificar atitudes relacionadas ao } \\
\text { consumo e às preferências alimentares. }\end{array}$ & $\begin{array}{l}\text { Os escolares apresentaram preferência por sucos industrializados e massas. O } \\
\text { consumo de frutas e hortaliças foi maior na frequência semanal. A maioria dos } \\
\text { escolares preferia fazer refeições com televisão ligada e os meninos relataram } \\
\text { sempre desejar experimentar alimentos anunciados nos intervalos comerciais. } \\
\text { Relataram que os alimentos mais solicitados por eles para que seus pais } \\
\text { comprassem eram salgadinhos industrializados, guloseimas e bolachas doces. }\end{array}$ \\
\hline
\end{tabular}

Fonte: Vieira MLR, Oliveira JCS, Ana Paula de Queiroz Mello APQ, 2015 - 2018.

REAS/EJCH | Vol.Sup.33 | e809 | DOI: https://doi.org/10.25248/reas.eXX.2019 Página 4 de 7 
Sabe-se que a infância é um período importante e decisivo para a formação do hábito alimentar. Nesta fase, as escolhas alimentares e a prática de atividade física são estabelecidas. Com isso, é fundamental a introdução de hábitos alimentares saudáveis e atividade física regular, com o objetivo de garantir que a criança desenvolva um estilo de vida saudável perpetuando-o na vida adulta (DURÉ ML et al. 2015)

De acordo com um estudo recente, apenas, 25\% das mães de crianças de 6 meses à 1 ano apresentaram introdução alimentar adequada. Mais de $40 \%$ das crianças consumiam alimentos ultraprocessados (AUPs), sendo as bolachas recheadas / chocolates / doces $(21,8 \%)$, seguida de bebidas doces $(20,0 \%)$ e macarrões instantâneos / salgadinhos de pacote / biscoitos salgados (18,5\%) os mais prevalentes. As crianças que não foram amamentadas apresentaram maior consumo de AUPs em comparação às que foram amamentadas (RELVAS GRB et al., 2018).

Outro estudo relata que escolares apresentaram o consumo à base de carne e arroz com feijão, e baixa ingestão de frutas, vegetais e leites. A preferência por industrializados se mostrou evidente pela escolha de massas, sucos artificiais, refrigerantes, achocolatado e guloseimas no geral. Um fato destacado pelos escolares foi que a principal fonte de informação é os pais, seguida pelos professores, e em terceiro lugar, a televisão (SOARES BR et al.,2017).

A televisão veicula propaganda que estimula o consumo de alimentos não saudáveis pelas crianças por meio da associação de personagens de desenhos infantis. Além da influência dos meios de comunicação de massa na escolha das preferências alimentares das crianças e dos pais, pode-se constatar a presença de pessoas autônomas ou funcionários da escola vendendo produtos industrializados e guloseimas na porta das creches. Nesse caso, mesmo com a oferta da merenda escolar gratuita, as crianças são induzidas à ingestão de produtos industrializados, já que estes estão facilmente acessíveis na escola e não há restrição do seu consumo (BENTO IC et al., 2015; FECHINE ADL et al., 2015).

Considerando que a publicidade alimentar é um importante determinante na escolha alimentar, não só as crianças, mas até mesmo os pais e cuidadores se rendem ao poder da televisão. Em vez de comerem a refeição à mesa, as famílias passaram a se reunir em frente à TV enquanto se alimentam. Nesse sentido, as indústrias de alimentos têm ampliado seus produtos, incluindo as mais variadas técnicas de marketing, com adoção de estratégias de promoção que incluem aspectos de facilidade e praticidade para o consumidor (FECHINE ADL et al., 2015).

O diálogo entre pais e filhos deve ser incentivado, principalmente quando se trata de questões relacionadas à influência das propagandas nas escolhas das crianças. Limitar o tempo de televisão, escolher programação infanto-juvenil com cunho educativo relacionado aos hábitos de vida saudável, evitando o acesso à programação abusiva que visa venda e lucro que se aproveitam da imaturidade da criança, característica desta fase de vida, devem ser incentivadas (DURÉ ML et al., 2015).

Na infância, as crianças têm como modelo os pais ou cuidadores. Diante da transição nutricional ocorrida no último século, destaca-se que a infância passou a ser contemplada como um estágio que começou a ser ameaçada por patologias contemporâneas associadas à má alimentação. Sendo que dietas inadequadas dos pais têm sido alguns dos principais responsáveis pelo aumento da prevalência da obesidade infantil, no qual, os pais e os outros membros da família, geralmente, estabelecem um ambiente partilhado em que o convívio pode ser propício à alimentação excessiva e/ou a um estilo de vida sedentário. Neste contexto, comem em excesso, muito rapidamente ou ignoram os sinais internos de saciedade, e oferecem um exemplo ruim às crianças. Em contrapartida, mães que selecionam preferencialmente os alimentos baseadas em critérios de qualidade e não apenas no sabor, oferecem refeições mais saudáveis às suas famílias (LINHARES et al., 2016).

Outro estudo revelou que o envolvimento, por parte dos avós na vida dos netos, implica em mudanças que atingem todos os membros da família, exigindo reformulações de regras e adaptações dos papéis desempenhados por cada membro do grupo familiar. Neste sentido, é importante ressaltar que seria errôneo pensar que tanto o pai quanto a mãe possuem papéis únicos ou iguais, contudo apesar das mudanças ocorridas na sociedade, existem atribuições que ainda são distintas para cada progenitor. De uma forma geral, 
constatou-se um modelo tradicional de papéis, para as mães coube o papel de ter maior participação na educação dos filhos e ser a maior provedora de carinho e afeto, e para os pais coube o papel do exercício da autoridade, do ensino e da disciplina (LINS ZMB et al., 2015).

Um aspecto relevante encontrado foi que apesar dos pais perceberem que os alimentos industrializados não são saudáveis para as crianças, é comum a oferta sistemática desses tipos de gêneros alimentícios em casa. Por conseguinte, o discurso dos pais apresenta-se ambíguo, pois mesmo sabendo que é errado, continuam ofertando alimentos não saudáveis aos filhos. Com isso, os alimentos mais consumidos são produtos energéticos de alta densidade nutricional ricos em gorduras, açúcares e sódio, pobres em fibras alimentares que podem causar doenças. (FECHINE ADL et al., 2015; BENTO IC et al., 2015).

Um exemplo de intervenção envolvendo a família é o Programa denominado Healthy Home Offerings via the Meal time Environment (HOME Plus), que foi criado para prevenir a obesidade entre crianças de 8 a 12 anos, com a participação dos pais. As intervenções foram compostas de dez sessões com duração de duas horas cada, e cinco telefonemas motivacionais com o objetivo de promover hábitos alimentares saudáveis e o aumento do consumo de refeições em família. Após as intervenções, houve melhora no consumo de frutas, legumes e verduras. Além disso, foi observado maior percepção em relação ao tamanho das porções, maior interesse dos filhos em experimentar novos alimentos e a presença de momentos agradáveis em família, envolvendo alimentação (FLATTUM C et al., 2015).

No que concerne a fatores de influência na seleção de alimentos por parte dos responsáveis, o preço se mostra como fator importante na tomada de decisões de compras, que pode afetar a qualidade da dieta. Limitações econômicas para a compra de alimentos, como as vivenciadas por famílias de baixa renda, regem a dietas com baixo consumo em frutas, legumes e verduras, e de alta carga energética, em relação aos cereais processados, óleos e açúcares (BENTO IC et al., 2015).

\section{CONSIDERAÇÕES FINAIS}

Podemos observar que vários fatores podem influenciar a formação do hábito alimentar e consequentemente, o estado nutricional das crianças, sobretudo verificamos que é importante que os pais tenham conhecimento das suas responsabilidades além de avaliar o impacto da mídia, cantina escolar, substituição de atividades físicas por atividades sedentárias e até mesmo a interferência de outros membros da família na formação de hábitos de saúde das crianças. Mesmo com a implantação das estratégias de saúde pública, no que diz respeito à alimentação e estilo de vida, ainda se faz necessário à promoção de ações de caráter educativo em diversos setores da sociedade. Além da família e da escola, as equipes de saúde multidisciplinares possuem parcelas significativas na disseminação de informações importante às quais servirão como base para a qualidade de vida da criança bem como de sua família.

\section{REFERÊNCIAS}

1. BENTO IC, et al. Alimentação saudável e dificuldades para torná-la uma realidade: percepções de pais/responsáveis por pré-escolares de uma creche em Belo Horizonte/MG, Brasil. Ciência \& Saúde Coletiva, 2015; 20(8): $2389-2400$.

2. CARVALHO ARM, et al. Sobrepeso e Obesidade e alunos de 6 - 10 anos de Escola Estadual de Umuarama / PR. Arquivos de Ciências da Saúde da UNIPAR, 2017; 21(1).

3. DURÉ ML, et al. A obesidade infantil: um olhar sobre o contexto familiar, escolar e da mídia. Revista de Epidemiologia e Controle de Infecção, 2015; 5(4) 91-196.

4. FECHINE ADL, et al. Percepção de pais e professores sobre a influência dos alimentos industrializados na saúde infantil. Revista Brasileira em Promoção da Saúde, 2015; 28(1) 16-22.

5. FERNANDES C, et al. Revisão integrativa sobre instrumentos de avaliação de consumo alimentar em crianças em idade escolar. Cogitare Enfermagem, 2017; 22(4).

6. FLATTUM C, et al. HOME Plus: Program design and implementation of a family-focused, community-based intervention to promote the frequency and healthfulness of family meals, reduce children's sedentary behavior, and prevent obesity. International Journal of Behavioral Nutrition and Physical Activity. 2015; 12(1).

7. FLORINDO AA, et al. Promoção da atividade física e da alimentação saudável e a saúde da família em municípios com academia da saúde. Revista Brasileira de Educação Física e Esporte. 2016; 30(4) 913-924. 
8. HARRISON ME, et al. Systematic review of the effects of family meal frequency on psychosocial outcomes in youth. Canadian Family Physician. 2015; 61(2) e96-e106.

9. INSTITUTO BRASILEIRO DE GEOGRAFIA E ESTATÍSTICA (IBGE). Pesquisa de orçamentos familiares 2008-2009: despesas, rendimento e condições de vida. Rio de Janeiro: Instituto Brasileiro de Geografia e Estatística; 2010.

10. JAIME PC, et al. Influência familiar no consumo de bebidas açucaradas em crianças menores de dois anos. Revista de Saúde Pública. 2017; 51 1-9.

11. LINHARES FMM, et al. Obesidade infantil: influência dos pais sobre a alimentação e estilo de vida dos filhos. Temas em Saúde; 2016 16(2).

12. LINS ZMB, et al. O papel dos pais e as influências externas na educação dos filhos. Revista da SPAGESP. 2015; 16(1) 43-59.

13. NASCIMENTO MMR, et al. Parents' perception of health-related quality of life in children and adolescents with excess weight. Jornal de Pediatria. 2016; 92(1) 65-72.

14. RELVAS GRB, et al. Ultra-processed food consumption among infants in primary health care in a city of the metropolitan region of Sao Paulo, Brazil. Jornal de pediatria. 2018.

15. SOARES BR, et al. Atitudes relativas ao consumo alimentar de escolares da zona sul de São Paulo/SP. Disciplinarum Scientia| Saúde, 2018; 18(2) 323-337.

16. SPARRENBERGER K, et al. Consumo de alimentos ultraprocessados entre crianças de uma Unidade Básica de Saúde. Jornal de Pediatria, 2015; 91(6).

17. TAVARES MG, et al. Obesidade: fatores de risco para doença cardiovascular na infância. Revista Hospital Universitário Pedro Ernesto, 2016; 15(2) 99-105.

18. WORLD HEALTH ORGANIZATION (WHO). 2019. In: World Health Organization. Global strategy on diet, physical activity and health: childhood overweight and obesity. Geneva: Diet Physical Activity. Disponível em: http://www. who.int/dietphysicalactivity/childhood/en. Acesso em: 4 abr 2019. 